\title{
Bedside myocardial perfusion assessment with contrast echocardiography
}

\author{
Sam Orde ${ }^{1 *}$ and Anthony McLean ${ }^{1,2}$
}

\begin{abstract}
This article is one of ten reviews selected from the Annual Update in Intensive Care and Emergency medicine 2016. Other selected articles can be found online at http://www.biomedcentral.com/collections/ annualupdate2016. Further information about the Annual Update in Intensive Care and Emergency Medicine is available from http://www.springer.com/ series/8901.
\end{abstract}

\section{Background}

Myocardial perfusion can be safely assessed at the bedside using contrast echocardiography. The contrast agents consist of tiny microbubbles (approximately $1-8 \mu \mathrm{m}$ in diameter), which remain in the systemic circulation for $\sim 3-5$ min after venous injection. Low intensity ultrasound imaging is required to prevent the microbubbles from being destroyed. Myocardial perfusion is assessed by destroying the microbubbles with a 'flash' of higher intensity ultrasound and then analyzing the replenishment rate as the microbubbles seep back into the myocardial circulation.

There is reasonable evidence that myocardial contrast perfusion echocardiography (MCPE) can help in the detection of coronary artery disease as well as having prognostic value over regional wall motion analysis. However, there are challenges in bringing it into everyday clinical use: the imaging is challenging and relatively complicated compared to standard echocardiography; the sensitivity and specificity are not $100 \%$; it remains an 'off-label' use of contrast echocardiography; and there are safety issues to consider. It has been investigated for more than 25 years and yet still has not made it into main-steam cardiac evaluation.

* Correspondence: sam.orde@hotmail.com

${ }^{1}$ Nepean Hospital, Intensive Care Unit, 2747 Sydney, NSW, Australia Full list of author information is available at the end of the article
One area of considerable interest and future potential is in critically ill patients who have raised cardiac enzymes, especially troponins, with or without electrocardiogram (EKG) abnormalities or regional wall motion abnormalities, in whom the diagnosis of ischemia needs to be addressed. Examples include Takotsubo's or septic cardiomyopathy. Investigation with angiography or further imaging may be detrimental in patients with acute renal failure or bleeding risk and there are dangers associated with unnecessary transfer. It is not suggested that MCPE would take the place of angiography or other investigations assessing myocardial perfusion, but potentially MCPE could identify patients (or at least triage them) who have normal myocardial perfusion yet abnormal troponins, EKGs and have regional wall motion abnormalities. In addition, there are exciting implications for the future use of microbubble contrast in terms of drug and gene delivery.

\section{Contrast echocardiography agents}

Echocardiography imaging in the critically ill can be frustratingly difficult at times. Contrast echocardiography agents were originally designed to help improve endocardial border definition, known as left ventricle opacification, as well as to enhance Doppler signals. Their use can prevent non-diagnostic studies from being inconclusive, particularly in the critically ill [1]. These contrast agents were originally described in the 1960s [2] and further development in the 1980s and '90s saw specific contrast agents designed to remain in the systemic circulation after venous injection, as well as ultrasound imaging enhancement techniques developed (such as harmonic imaging) to enhance left ventricular opacification [3-5].

The contrast agents consist of microbubbles containing a hemodynamically inert gaseous core (e.g., octafluoropropane, sulfur hexafluoride) and a stabilizing outer shell (e.g., lipid, albumin or biopolymer), which oscillate under the influence of ultrasound waves [6]. 
Similar to agitated saline, now in use for over 35 years to determine cardiac and intrapulmonary shunts, these contrast echocardiography microbubbles form multiple small liquid-air interfaces whose boundaries have a high acoustic impedance mismatch resulting in enhanced ultrasound reflection. A major difference of contrast microbubbles compared to saline bubbles is the size, with the bubbles small enough $(1-8 \mu \mathrm{m})$ to traverse the pulmonary capillaries in order to enter the systemic circulation. Saline bubbles are typically $50-90 \mu \mathrm{m}$ diameter and are destroyed as they pass into the pulmonary capillaries.

Microbubbles require specific 'activation' to be effective (different methods are required for different agents). Injected intravenously, they cross the pulmonary circulation into the system circulation. With similar behavior and rheology to red blood cells (RBCs) [7], they remain entirely within the vascular compartment and last in the circulation for approximately $3-5 \mathrm{~min}$ before they burst and lose their ability to produce ultrasound backscatter. Once the microbubbles are destroyed, the shell is metabolized by fatty acid metabolism if made of lipid (such as with Definity [BMS, Billerica, MA]), or by the reticuloendothelial system. The inert gas is not metabolized and simply escapes from the lungs [8].

There are various contrast agents available, each having slightly different compositions and gas cores (Table 1). Different countries have different agents available. The first generation contrast agents, developed at the end of the $20^{\text {th }}$ century, have a lipid shell with an air core, are soluble and are able to pass through the pulmonary circulation but lose their echogenicity and dissolve rapidly. The second generation contrast agents were then developed and have high-molecular weight gaseous cores, are less soluble than air, with stabilizing lipid or biopolymer shells and remain more stable under the ultrasound field and, therefore, have an increased lifespan in the circulation [9]. These preparations include the standard contrast agents used today: Definity, Optison (GE healthcare, Chalfont St Giles, UK) and Sonovue (Bracca, Milan, Italy). Third generation agents include those specifically used for researchbased activities, specialized imaging or therapeutic purposes [8].

\section{Effect of ultrasound on contrast agents}

Specific imaging techniques and software are required to perform MCPE to take advantage of the different ultrasound reflection properties of the contrast microspheres versus soft tissue. When ultrasound interacts with the microbubbles they oscillate and this effect is dependent on the ultrasound acoustic pressure as well as the shell and core gas properties of the agent. Ultrasound acoustic pressure is described as the 'mechanical index' and corresponds to the power output of the scanner [10]. With standard 2D echocardiography imaging the mechanical index is $\sim 1.4$; however, at this level the microspheres would oscillate to such a degree that they would burst and be destroyed. Therefore low mechanical index $(<0.2)$ imaging is used with contrast imaging.

The oscillation effect of contrast echocardiography under low mechanical index ultrasound means the ultrasound reflections are different for microbubbles compared to soft tissue. This difference can be harnessed to enhance contrast versus tissue differentiation when imaging: microbubbles reflect ultrasound in a non-linear format compared to tissue, which reflects ultrasound in a linear manner. Non-linear reflection means the sound waves are reflected not only at the frequency of the original ultrasound wave but also at higher, harmonic frequencies. Soft tissue, however, produces fewer harmonics, hence reflection of the ultrasound waves in a more linear fashion. There are different methods used

Table 1 Contrast echocardiography agents

\begin{tabular}{|c|c|c|c|c|c|}
\hline Classification & Gas core & Shell & Trade name & Bubble size $(\mu \mathrm{m})$ & Comments \\
\hline \multirow[t]{3}{*}{ First generation } & Air & Albumin & Albunex & $2-8$ & No longer made \\
\hline & Air & Palmitic acid/galactose & $\begin{array}{l}\text { Levovist (Schering, } \\
\text { Kelinworth, NJ) }\end{array}$ & $2-8$ & Non-cardiac use mainly \\
\hline & Air & D-galactose & $\begin{array}{l}\text { Echovist (Berlex, Lachine, } \\
\text { Quebec City, Canada) }\end{array}$ & $2-8$ & $\begin{array}{l}\text { First commercially available } \\
\text { agent }\end{array}$ \\
\hline \multirow[t]{3}{*}{ Second generation } & Octafluoropropane $\left(\mathrm{C}_{3} \mathrm{~F}_{8}\right)$ & Albumin & $\begin{array}{l}\text { Optison (GE healthcare, } \\
\text { Chalfont St Giles, UK) }\end{array}$ & $1-10$ & $\begin{array}{l}\text { Available in USA, Europe, } \\
\text { South America }\end{array}$ \\
\hline & Octafluoropropane $\left(\mathrm{C}_{3} \mathrm{~F}_{8}\right)$ & Lipid & Definity (BMS, Billerica, MA) & $1-10$ & $\begin{array}{l}\text { Available in USA, Europe, } \\
\text { South America, Canada, } \\
\text { Australasia }\end{array}$ \\
\hline & Sulfur hexafluoride $\left(\mathrm{SF}_{6}\right)$ & Lipid & Sonovue (Bracca, Milan, Italy) & $1-10$ & $\begin{array}{l}\text { Available in Europe and } \\
\text { USA (known as Lumason) }\end{array}$ \\
\hline
\end{tabular}

NB: The list does not include every available contrast agent worldwide and the accuracy of the 'comments' section may change but is up to date at time of writing to the best of the authors' knowledge 
by various vendors to take advantage of the specific reflection properties for tissue vs contrast microbubbles, including: pulse inversion, power modulation and coherent contrast imaging to reduce the soft tissue linear reflections of the fundamental frequency [11].

\section{Myocardial perfusion imaging}

In the 1990s, initial studies in animals, subsequently validated in humans, investigated the hypothesis that myocardial blood flow could be assessed with contrast echocardiography by destroying the contrast microbubbles with a 'flash' of high diagnostic intensity ultrasound and then assessing the rate of replenishment of the microbubbles into the myocardium [12-14]. The replenishment is assessed by the change in intensity or brightness in a 'region of interest' (ROI). The microbubbles behave like $\mathrm{RBCs}$, hence the theory that any change in signal intensity represents a change in myocardial blood flow.

With normal myocardial blood flow, $90 \%$ of the coronary circulation resides within the myocardial capillaries and RBCs travel at approximately $1 \mathrm{~mm} / \mathrm{s}$ at rest. After destruction of the contrast the signal intensity is anticipated to return to normal after approximately 5-7 cardiac cycles [13] (Fig. 1). During stress or exercise where vasodilation and increased capillary blood flow are present, the rate of return of signal intensity is faster: approximately $2-3$ cardiac cycles. The rate of microbubble replenishment can be assessed qualitatively (as seen in Fig. 1), but also quantitatively by reviewing the change in signal intensity over time in a specific ROI (Fig. 2). Myocardial blood flow is considered the product of plateau signal intensity and rate of replenishment (Fig. 3). The concept being that the slower the rate of replenishment and lower the plateau signal intensity, the poorer the myocardial blood flow.

\section{Safety profile}

The use of contrast echocardiography, extensively investigated in several large multicenter trials [15-17], has been found to be well-tolerated and safe in both noncritically ill and critically ill patients [18]. 'Black-box' warnings were issued by the US Food and Drug Administration (FDA) in 2007 but these were downgraded within 12 months. The current FDA recommendations state that if a patient has an unstable cardiopulmonary condition or pulmonary hypertension (the severity is not stated), the patient should have cardiorespiratory monitoring for $30 \mathrm{~min}$ after contrast agent administration [19]. In the United States, echocardiography laboratories are not accredited unless they have the ability to perform contrast echocardiography [20].

Side effects are rare and include headache, flushing or back pain. These symptoms are usually relieved on cessation of contrast agent administration. There is a 1:10,000 chance of an anaphylaxis type reaction (considered secondary to the microbubble shell and possibly non-IgE related) [9]. Contraindications include previous hypersensitivity to contrast agents or to blood products (e.g., albumin), severe pulmonary hypertension and cardiac right-to-left or bidirectional shunts. These last two contraindications are under debate and evidence exists of the safety in these conditions, whereas there are only case reports of harm with recent use of ultrasound contrast $[16,21]$.

We consider an individualized approach of risk versus benefit is required for MCPE. Important requirements

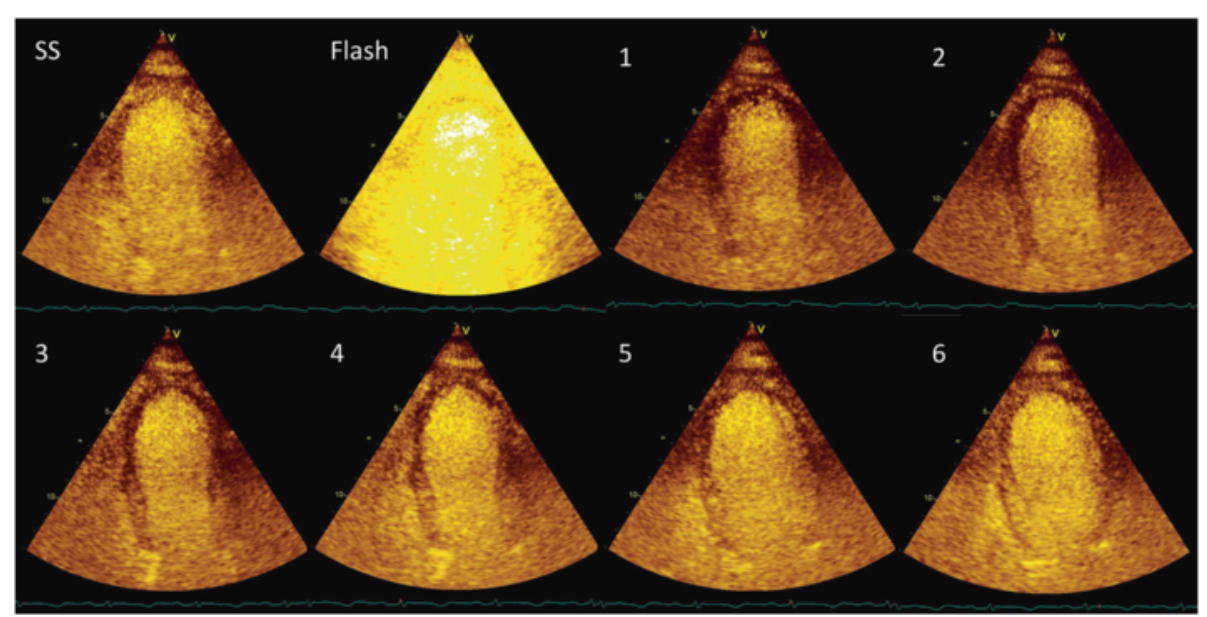

Fig. 1 Normal myocardial contrast perfusion echocardiograph: qualitative assessment. Ultrasound contrast infused until steady state achieved (SS - steady state). A 'flash' of high mechanical index ultrasound destroys the contrast microbubbles within the imaging beam. Assessment of myocardial perfusion is then made as the microbubbles return to the myocardium over subsequent cardiac cycles (1-6). Normal replenishment occurs over 5-6 cardiac cycles at rest, 2-3 cardiac cycles with stress 


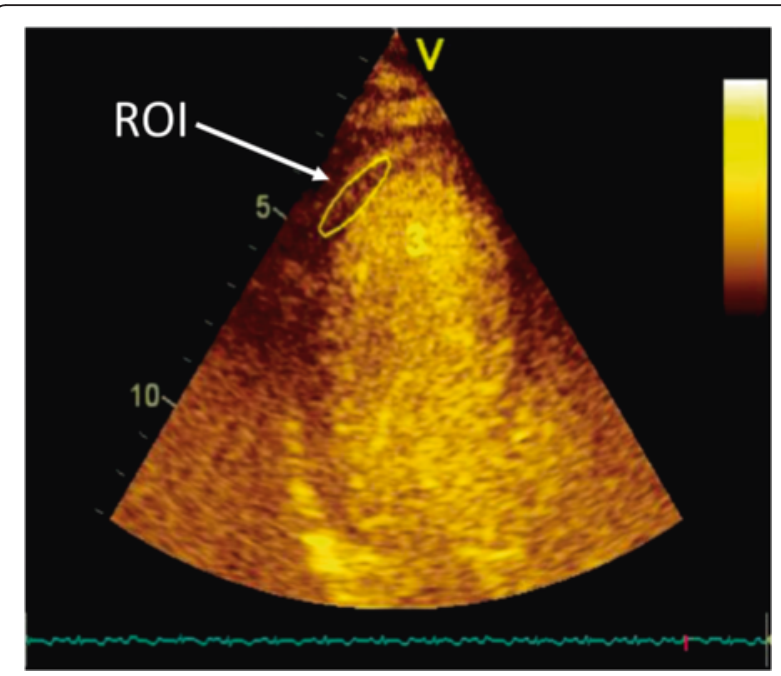

Fig. 2 Qualitative assessment of myocardial perfusion involves specification of a region of interest (ROI) classically corresponding to individual left ventricular myocardial segments

include expertise to perform and interpret the procedure, and the study should be performed in an environment with appropriate monitoring and resuscitation facilities.

\section{Applications in the critically ill}

\section{Recognition of acute coronary artery disease}

The diagnosis of acute coronary syndromes (ACS) in the intensive care unit (ICU) can be challenging. Critically ill patients with ischemic heart disease are at greater risk during times of stress and the classic history of central crushing chest pain can be absent as a result of acute illness, sedation and/or mechanical ventilation. Troponin elevation, EKG and regional wall motion abnormalities (RWMA) are frequently seen in conditions other than myocardial infarction [22], for example Takotsubo's and septic cardiomyopathy amongst many other causes [23]. In addition, investigating for possible ACS with angiography or single photon emission computed tomography (SPECT) can be dangerous due to the inherent risks of patient transport, contrast-induced nephropathy, radiation, access issues, anticoagulation, and delays in diagnosis. Cost and access to suitable angiographic facilities may be issues in some ICUs. Potentially, MCPE could help identify patients with ACS at the bedside in the ICU, not to replace further imaging, but rather as a triage tool or simply to add confidence to the physician's clinical acumen [24].

MCPE has been compared to SPECT, the most widely used perfusion technique for assessment of coronary artery disease. In several studies for detection of coronary artery disease, MCPE has shown excellent concordance
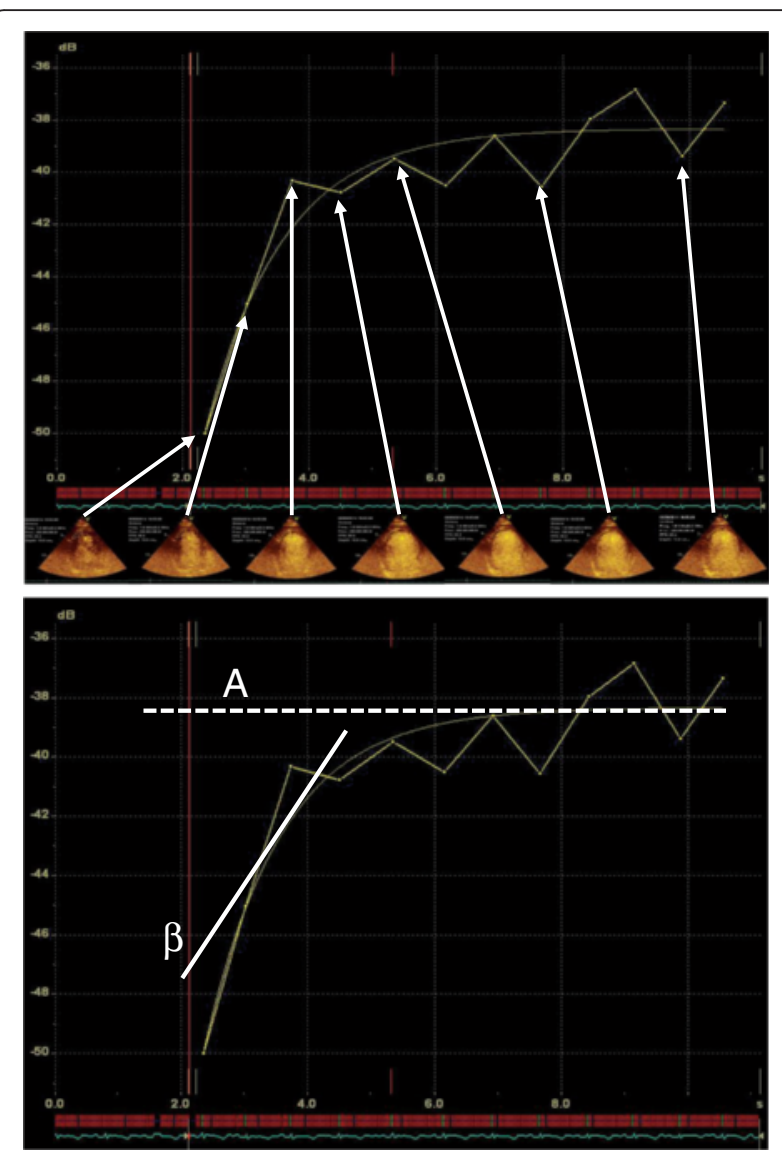

Fig. 3 Myocardial contrast perfusion echocardiography (MCPE) quantitative assessment. Regions of interest (ROI) are defined and rate of change in signal intensity assessed at end-diastolic frames. The plateau signal intensity $(A)$ is considered to represent the myocardial capillary blood volume. The rate of replenishment ( $\beta$ ) of the microbubbles is considered as the velocity of blood. The product of $A \times \beta$ is considered to represent the myocardial blood flow

(81 \% [76.4-85.6]) [25]. A meta-analysis indicated a higher sensitivity for MCPE than for SPECT and no difference was found for specificity [26]. Various clinical studies have used MCPE to quantify myocardial blood flow, trying to differentiate coronary artery ischemia from not significantly occluded coronary arteries. Senior et al. reported that MCPE could differentiate ischemic from non-ischemic cardiomyopathy (defined as $<50 \%$ coronary artery stenosis) with a specificity of $89 \%$ and sensitivity of $91 \%$ [27].

Microvascular versus macrovascular function assessment Microvascular dysfunction has been proposed in a number of cardiac conditions such as Takotsubo's [28] and septic cardiomyopathy [29] amongst others. Whether the microvascular dysfunction is a primary cause of secondary phenomena is not known. Abdelmoneim et al 
performed MCPE in 9 patients with angiographically confirmed Takotsubo's syndrome and were able to show reduced perfusion in the myocardium with a $71 \%$ concordance with areas of RWMA [28]. It is suggested that the microvasculature in the endocardial regional has the lowest flow reserve and is more susceptible to ischemia than the epicardium possibly due to the larger epicardially placed coronary arteries [30]. Therefore, with microvascular disorders there may be a reduction in the endocardial myocardium to a greater extent than in the epicardial myocardium (Fig. 4).

\section{Possible future roles for contrast echocardiography}

Advances in contrast microbubble formulations, imaging and post-processing analysis, indicate that the future for contrast echocardiography may include imaging of macro and microvasculature elsewhere in the body as well as targeted drug and/or gene delivery.

\section{Contrast-enhanced ultrasound}

Using contrast agents in a similar manner to MCPE, non-invasive and bedside perfusion assessment of organs may be possible. Schneider et al. suggested that assessing renal cortical perfusion with contrast is feasible and well-tolerated in the ICU population and that possibly a decrease in renal perfusion may occur within $24 \mathrm{~h}$ of surgery in patients at risk of acute kidney injury (AKI) [31]. These techniques are relatively unexplored at this time and although they hold promise, do demonstrate significant heterogeneity and the results remain unpredictable [32]. Further investigation is certainly warranted.

\section{Targeted drug delivery}

The property of contrast microbubbles bursting under the effect of ultrasound can be used to target drug delivery. Drugs can be attached to microbubbles by a variety of methods [10] and as long as the site is accessible to ultrasound, a burst of high mechanical index ultrasound may be able to locally deliver the drug, such as thrombolysis. Transfer of genetic material has also been suggested and has been shown to be safe and more specific than viral vectors for cDNA delivery [33].

\section{Conclusion}

The use of contrast echocardiography in the critically ill is safe compared to other contrast agents, feasible at the bedside and has the potential to rescue undiagnostic echocardiograms. Although the agents are only indicated for left ventricular opacification, the off-label use of MCPE holds promise as being a potential method to assess myocardial perfusion at the bedside. The technology has been available for over two decades and is yet to find a place in regular clinical practice, but as a result of ever evolving sophistication of microbubble agents, software and hardware still holds considerable promise. The utility of MCPE in the ICU has not been extensively considered to date but potentially may have a role in the challenging arena of accurate and timely diagnosis of ACS in the critically ill.

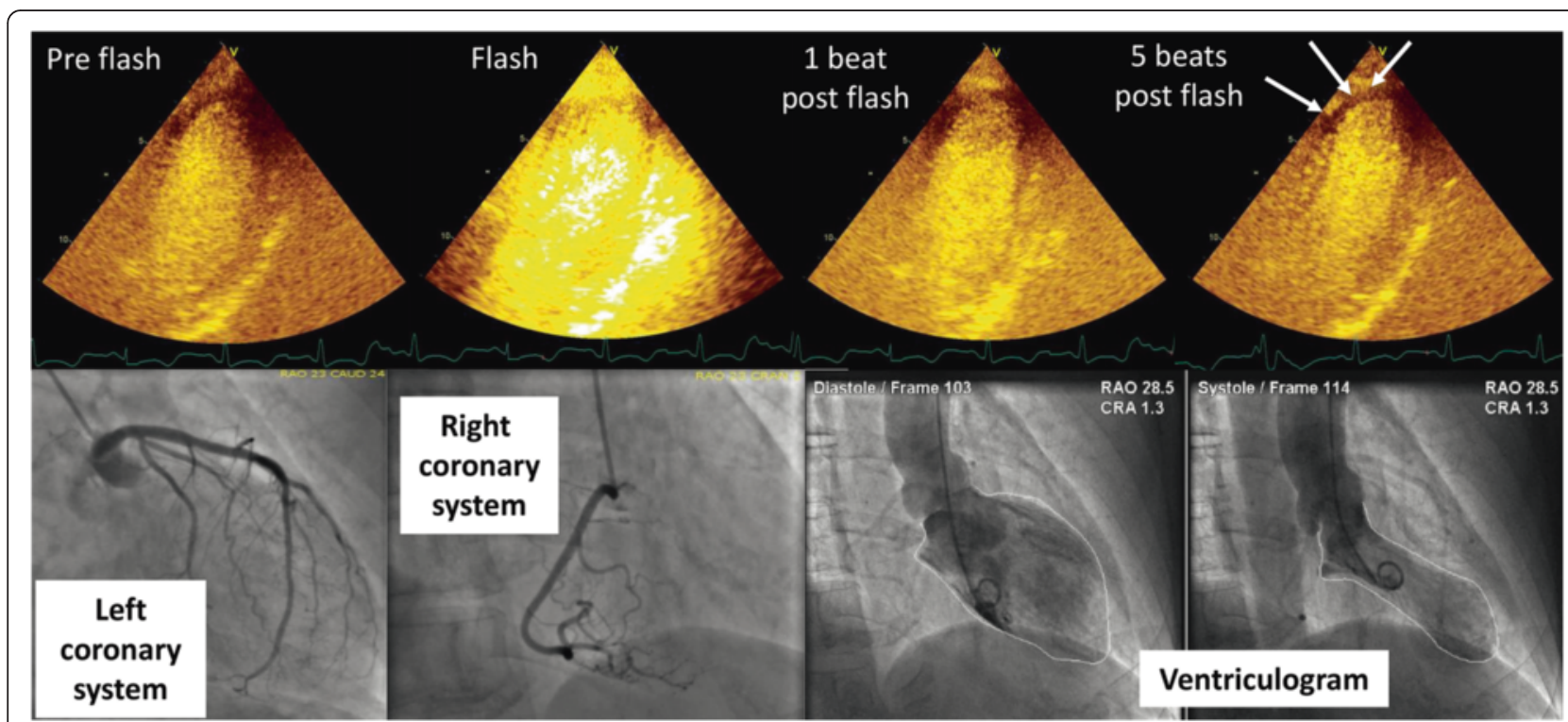

Fig. 4 Takotsubo's cardiomyopathy with microvascular dysfunction (arrows). Endocardial perfusion defect shown at 5 beats post flash in the apical region where transient apical hypokinesis was visualized. Coronary angiography confirmed normal vasculature and left ventriculography demonstrated apical ballooning 


\section{Competing interests}

The authors declare that they have no competing interests.

\section{Authors' contributions}

Both authors read and approved the final manuscript.

\section{Declarations}

Open access publication of the article was funded by the authors.

\section{Author details}

${ }^{1}$ Nepean Hospital, Intensive Care Unit, 2747 Sydney, NSW, Australia.

${ }^{2}$ University of Sydney, Sydney Medical School, Sydney, Australia.

\section{Published online: 15 March 2016}

\section{References}

1. Kurt M, Shaikh KA, Peterson L, et al. Impact of contrast echocardiography on evaluation of ventricular function and clinical management in a large prospective cohort. J Am Coll Cardiol. 2009;53:802-10.

2. Gramiak R, Shah PM, Kramer DH. Ultrasound cardiography: contrast studies in anatomy and function. Radiology. 1969;92:939-48.

3. Keller MW, Feinstein SB, Watson DD. Successful left ventricular opacification following peripheral venous injection of sonicated contrast agent: an experimental evaluation. Am Heart J. 1987:114:570-5.

4. Feinstein SB, Cheirif J, Ten Cate FJ, et al. Safety and efficacy of a new transpulmonary ultrasound contrast agent: initial multicenter clinical results. J Am Coll Cardiol. 1990;16:316-24.

5. Lindner JR, Dent JM, Moos SP, et al. Enhancement of left ventricular cavity opacification by harmonic imaging after venous injection of Albunex. Am J Cardiol. 1997;79:1657-62.

6. Postema M, van Wamel A, Ten Cate FJ, de Jong N. High-speed photography during ultrasound illustrates potential therapeutic applications of microbubbles. Med Phys. 2005;32:3707-11.

7. Keller MW, Segal SS, Kaul S, Duling B. The behavior of sonicated albumin microbubbles within the microcirculation: a basis for their use during myocardial contrast echocardiography. Circ Res. 1989;65:458-67.

8. Platts DG, Fraser JF. Contrast echocardiography in critical care: echoes of the future? A review of the role of microsphere contrast echocardiography. Crit Care Resusc. 2011:13:44-55.

9. Seol S-H, Lindner JR. A primer on the methods and applications for contrast echocardiography in clinical imaging. J Cardiovasc Ultrasound. 2014;22:101-10.

10. Pathan F, Marwick TH. Myocardial perfusion imaging using contrast echocardiography. Prog Cardiovasc Dis. 2015;57:632-43.

11. Seol S-H, Davidson BP, Belcik JT, et al. Real-time contrast ultrasound muscle perfusion imaging with intermediate-power imaging coupled with acoustically durable microbubbles. J Am Soc Echocardiogr. 2015;28:718-726.e2

12. Jayaweera AR, Edwards N, Glasheen WP, et al. In vivo myocardial kinetics of airfilled albumin microbubbles during myocardial contrast echocardiography. Comparison with radiolabeled red blood cells. Circ Res. 1994:74:1157-65.

13. Wei K, Jayaweera AR, Firoozan S, et al. Quantification of myocardial blood flow with ultrasound-induced destruction of microbubbles administered as a constant venous infusion. Circulation. 1998;97:473-83.

14. Vogel R, Indermühle A, Reinhardt J, et al. The quantification of absolute myocardial perfusion in humans by contrast echocardiography: algorithm and validation. J Am Coll Cardiol. 2005;45:754-62.

15. Main ML, Hibberd MG, Ryan A, et al. Acute mortality in critically ill patients undergoing echocardiography with or without an ultrasound contrast agent. JACC Cardiovasc Imaging. 2014;7:40-8.

16. Abdelmoneim SS, Bernier M, Scott CG, et al. Safety of contrast agent use during stress echocardiography in patients with elevated right ventricular systolic pressure: a cohort study. Circ Cardiovasc Imaging. 2010;3:240-8.

17. Wei K, Mulvagh SL, Carson L, et al. The safety of deFinity and Optison for ultrasound image enhancement: a retrospective analysis of 78,383 administered contrast doses. J Am Soc Echocardiogr. 2008;21:1202-6.

18. Putrino A, Platts DG. Contrast echocardiography in acutely unwell patients. J Am Soc Echocardiogr. 2015;28:844.

19. U.S. Food and Drug Administration. Postmarket Drug Safety Information for Patients and Providers. Information for Healthcare Professionals: Micro-bubble Contrast Agents (marketed as Definity (Perflutren Lipid Microsphere) Injectable
Suspension and Optison (Perflutren Protein-Type A Microspheres for Injection). 2008. Update FDA alert. http://www.fda.gov/Drugs/DrugSafety/Postmarket DrugSafetylnformationforPatientsandProviders/ucm125574.htm. Accessed Oct 2015

20. Mulvagh SL, Rakowski H, Vannan MA, et al. American society of echocardiography consensus statement on the clinical applications of ultrasonic contrast agents in echocardiography. J Am Soc Echocardiogr. 2008;21:1179-201.

21. Parker JM, Weller MW, Feinstein LM, et al. Safety of ultrasound contrast agents in patients with known or suspected cardiac shunts. Am J Cardiol. 2013:112:1039-45.

22. Lim W, Whitlock R, Khera $V$, et al. Etiology of troponin elevation in critically ill patients. J Crit Care. 2010;25:322-8.

23. Orde SR, Pulido JN, Masaki M, et al. Outcome prediction in sepsis: speckle tracking echocardiography based assessment of myocardial function. Crit Care. 2014;18:R149.

24. Orde SR, Huang SJ, McLean AS. 2015 ASE 26th Annual Scientific Sessions. Raised Troponin in the ICU: can real-time myocardial contrast echocardiography improve recognition of acute ischaemia in the critically ill? JASE. 2015:28:B2-B134. abst.

25. Senior R, Becher $\mathrm{H}$, Monaghan M, et al. Contrast echocardiography: evidence-based recommendations by European Association of Echocardiography. Eur J Echocardiogr. 2009;10:194-212.

26. Dijkmans PA, Senior $R$, Becher $H$, et al. Myocardial contrast echocardiography evolving as a clinically feasible technique for accurate, rapid, and safe assessment of myocardial perfusion: the evidence so far. J Am Coll Cardiol. 2006;48:2168-77.

27. Senior R, Janardhanan R, Jeetley P, Burden L. Myocardial contrast echocardiography for distinguishing ischemic from nonischemic first-onset acute heart failure: insights into the mechanism of acute heart failure. Circulation. 2005:112:1587-93.

28. Abdelmoneim SS, Mankad SV, Bernier M, et al. Microvascular function in Takotsubo cardiomyopathy with contrast echocardiography: prospective evaluation and review of literature. J Am Soc Echocardiogr. 2009:22:1249-55.

29. Ait-Oufella H, Bourcier S, Lehoux S, Guidet B. Microcirculatory disorders during septic shock. Curr Opin Crit Care. 2015:21:271-5.

30. Linka AZ, Sklenar J, Wei $K$, et al. Assessment of transmural distribution of myocardial perfusion with contrast echocardiography. Circulation. 1998;98:1912-20.

31. Schneider AG, Goodwin MD, Schelleman A, et al. Contrast-enhanced ultrasound to evaluate changes in renal cortical perfusion around cardiac surgery: a pilot study. Crit Care. 2013;17:R138.

32. Schneider AG, Goodwin MD, Schelleman A, et al. Contrast-enhanced ultrasonography to evaluate changes in renal cortical microcirculation induced by noradrenaline: a pilot study. Crit Care. 2014;18:653.

33. Bhattacharyya S, Senior R. The current state of myocardial contrast echocardiography: what can we read between the lines? Reply. Eur Heart J Cardiovasc Imaging. 2014;15:351-2. 